\title{
Analysis of quantum neural network learning ability
}

\author{
Yan-hua Zhong ${ }^{1}$, Chang-qing Yuan ${ }^{2}$ \\ ${ }^{1}$ Depart. Of Electronics and Information Technology, Jiangmen Polytechnic, Jiangmen 529090, China \\ ${ }^{2}$ Aviation University of Air Force, Changchun 130022, China
}

Received: 24 Oct. 2012, Revised: 8 Jan. 2013, Accepted: 11 Jan. 2013

Published online: 1 Jun. 2013

\begin{abstract}
This paper represents the spatial-temporal integration of neural signals from the Quantum Hamiltonian of the system of analysis of quantum neuron. A theoretical quantum neural network model is proposed by quantum neural model of wave functions. Quantum Turing machine operator in a single step has been constructed and the study algorithm has been presented. Simulation results show that quantum neuron has a strong ability to learn. Finally some other related problems about quantum neural computation have been discussed.
\end{abstract}

Keywords: quantum neural computation; quantum computation; quantum neural network; Turing machine operator

\section{Introduction}

The combination of Quantum theory and neural computation was proposed by Louisiana State University Professor Kak, in 1995. He published "On Quantum Neural Computing" and it first proposed the concept of quantum neural computing. Motivation of research on quantum neural networks: first, quantum effect is related to human brains and plays an important role accordingly. As early as 1989, Penrose discussed the relationship of quantum phenomena and human consciousness, and pointed out that solving problem is a prerequisite to final settlement of the consciousness of quantum measurement [2]; Perus (1996) pointed out that quantum wave function collapse is very similar to a nerve in human brains and memory model reconstruction [3]. Hartford University of Gould further proved that it has glass ER (Bohr) of quantum process of ontology on the explaination and perception. Manager of brain process of full sexual theory also has mathematics structure. These two processes of dynamic equation contain a field, which is quantum potential or neural potential, basing on quantum potential of quantum process and neural potential of brain. Process of dynamic equation amazingly has similar of Department [4]. Second, because the quantum theory is a product of classical physics to the development of micro level, it has a characteristics of more general nature. Thus infer, quantum neural network should be the natural evolution of traditional neural computing systems, so the power of quantum computation will be used, upgrading the processing power of neural computation. These creative researches for quantum computation with neural computing provided useful support. In recent years, some scholars have proposed a variety of quantum neural algorithms and applications. This article is from the establishment of quantum neural model demonstration of quantum neural learning ability in the wave function.

\section{The Classical Neural network}

The first logical neuron model (i.e. M-P model) was developed by W.S. McCulloch and W.A. Pitts in 1943 [2]. It described the fundamental functions and structures of a neural cell reporting that a neuron will fire an impulse only if a threshold value is exceeded. The artificial neural cell is also called processing unit, nodal point or short time memory. The outputs from the outside circumstances or from the other neural cell construct the input vector $\left(x_{1}, x_{2}, \cdots, x_{n}\right)^{T}$. In here, $x_{i}$ is the input vector, the adjustable value $w_{i j}$ conjoined by two neural cells is called the weights or long time memory. All the weights conjoined with the neural cells construct the weights vector $W_{j}=\left(\omega_{j 1}, \omega_{j 2}, \cdots, \omega_{j n}\right)^{T}$ The computation

\footnotetext{
* Corresponding author e-mail: zhflowers@ 163.com
} 
process for neural cell is:

$$
y=f\left(\sum_{i=1}^{n} \omega_{i j} x_{i}-\theta_{j}\right)
$$

In this expression (1), $y$ is output, $f$ is the activation function that determines the output value. A simple choice for $f$ is the signal function $\operatorname{sig}(\cdot)$. In this case, the weights are used to calculate a weighted sum of the inputs. If it exceeds the threshold $\theta_{j}$, the output is 1 else the value of $y$ is -1 . But the McCulloch-Pitts neuron did not have mechanisms for learning. Based on biological evidences, D.O. Hebb suggested a rule to adapt the weights, that is, a learning rule [2]. This biological inspired procedure can be expressed in the following manner:

$$
\omega_{j}(t-1)=\omega_{j}(t)+\eta(d-y(t)) x_{j}
$$

Where $\omega_{j}(t-1)$ and $\omega_{j}(t)$ are adapted weights and initials weights respectively, $\eta$ is a real parameter to control the rate of learning and $d$ is the desired (know) output. This learning rule is called the perceptron model for a neuron. The learning typically occurs for example through training, or exposure to a known set of input/output data. The training algorithm iteratively adjusts the connection weights $\left\{\omega_{i j}\right\}$ analogous to synapses in biological nervous. These connection weights store the knowledge and are necessary to solve specific problems.

\section{Quantum model systems}

In practice, the most useful model for quantum computation is the Quantum Computational Network, also called Deutsch's model [5,9]. The basic information unit in this model is a qubit, which can be considered a superposition of two independent states $\mid 0>$ and $\mid 1>$, denoted by $|\varphi>=a| 0>+b \mid 1>$, where $a, b$ are complex numbers such that $|a|^{2}+|b|^{2}=1$, A composed system with $N$ qubits is described using $N=2^{n}$ independent states obtained through the tensor product of the Hilbert Space associated with each qubit.

The neuron of quantum perception machine model is formed by $n$ quanta which have $x_{i}$ distance between each other and is inlaid in the semiconductor. One quantum dot represents one neuron. Every ground state and first excited state is taken as calculation base, marks for $\mid 0>$ and $\mid 1>$. Quanta can be $n$ spin-particle which interactsor can be $n$ electrons which interacts though the electric doublets. The whole Hamiltonian operator is divided into two parts:

$$
\hat{H}=\hat{H}_{0}+\hat{H}^{t},
$$

Where $\hat{H}_{0}$ has nothing to do with time and corresponds to initial state; $\hat{H}^{t}$ relates with time and represents the time and space conformity for the system which being influenced by the environment. $\hat{H}_{0}$ also can be expressed in this way:

$$
\hat{H}_{0}=\sum_{i=1}^{n} \hat{H}_{i}+\sum_{i=1}^{n} \hat{H}_{i j}
$$

Here $\hat{H}_{i}$ is Hamiltonian operator of $i$ particle, and $\hat{H}_{i j}$ is coupled Hamiltonian operator of $i$ particle and $j$ particle, among them

$\hat{H}_{i}=\hbar \omega_{i} \hat{S}_{i Z}$,

$\hat{H}_{i j}=4 \hbar \Omega \hat{S}_{i Z} \hat{S}_{j Z}$,

Where $\hat{S}_{i Z}$ is $z$ component for the spin operator of $i$ spin-particle. $\Omega$ is the coefficient of coupling, and its dimension depends on the spin orientation as well as the distance between two particles. $\hat{H}_{0}$ is the opposite angles at $2 n$ dimension in Hilbert space base when two particles are close enough $(\Omega>0)$, the couple energy of the particles is $E>0$ corresponded $\mid 00>$ and $\mid 11>$ state and is $E<0$ corresponded $\mid 10>$ and $\mid 01>$ state. According to the difference of the state, level width of energy can be $\pm \hbar \Omega$. It can cause a particle turn over according to the spin state of another particle by the suitable selection of frequency pulse.

The time and space conformity for the neuron signal of the nervous system can be described with the Feynman path integral formulation of quantum mechanics as follows:

$$
\begin{aligned}
\mid \psi\left(x_{f}, T\right)>= & \int_{\left(x_{0}, 0\right)}^{\left(x_{f}, T\right)} D[x(t)] \times \\
& \exp \left(\frac{i}{\hbar} \int_{0}^{T} d t\left[\frac{1}{2} m \dot{x}^{2}-V(x)\right]\right) \mid \psi\left(x_{0}, 0\right)>,
\end{aligned}
$$

Here $\left|\psi\left(x_{0}, 0\right)\right\rangle$ is the input state of the quantum system at the time $t=0$ and $\mid \psi\left(x_{f} T\right)>$ is the output state at the time $t=T$. In this equality, $m$ is the mass, $h$ is Planck's constant, and $V(x)$ is the potential energy.

The interaction between Neuron and the environment as time develops, will lead to quantum dissipation and decoherence, and this has embodied the nonlinearity course for neuron. Supposed the environment state is represented with $\mid e>$, and the pair states are represented separately with $\mid e_{i}>$ and $\left|e_{i}^{\prime}\right\rangle$ in evolution state corresponded qubit state $\mid 0>$ and $\mid 1>$. The environment state is shown as follows:

$$
\begin{aligned}
& e_{ \pm}>=\frac{1}{2}\left(\left|e_{0}> \pm\right| e_{1}>\right) \\
& e_{ \pm}^{\prime}>=\frac{1}{2}\left(\left|e_{0}^{\prime}> \pm\right| e_{1}^{\prime}>\right) .
\end{aligned}
$$

Thus the general state of qubit $\alpha|0>+\beta| 1>$ and environment will change as:

$$
\begin{aligned}
& \mid e>(\alpha|0>+\beta| 1>) \\
& \quad \rightarrow\left[\left|e_{+}>I+\right| e_{-}>\hat{\sigma}_{3}+\left|e_{+}^{\prime}>\hat{\sigma}_{1}-\right| e_{-}^{\prime}>i \hat{\sigma}_{2}\right], \\
& \cdot(\alpha|0>+\beta| 1>)
\end{aligned}
$$


Here, $I$ is unit matrix and $\hat{\sigma}_{1}, \hat{\sigma}_{2}, \hat{\sigma}_{3}$ are three Pauli's matrixes. The four results shown at Expression (7) include all the interaction between a qubit and environment. That is to say, the error rate of a neural is $3 / 4$.

According to this model, the activation for each neuron with time can be described as:

$$
\psi_{i}(t)=\psi\left(x_{i}, t\right),
$$

$n$ neurons construct $n$ Hilbert space of degree of freedom in this nervous system; therefore the state of the change with time can be shown as:

$$
S(t)>=\left|\psi_{1}>\otimes\right| \psi_{2}>\otimes\left|\psi_{3}>\otimes \cdots\right| \psi_{n}>,
$$

Where $\left|\psi_{i}\right\rangle$ is quantum state $\psi, \otimes$ is tensor product. Generally, when the neurons is activated, it is presented with $\mid 1>$ (the spin is downward), and when the neurons is restrained, it is presented with $\mid 0>$ (the spin is upwards). So, the systematic state can be encoded with the binary quantum bit. After the time being discretized, Expression (8) is equivalent to the following one:

$$
\psi_{i}(t+1)=\sum_{j=1}^{n} G\left(x_{i}, x_{j}\right) \varphi_{j}(t),
$$

Or write it in this way: $\psi(t+1)=D(\psi(t))$, here $\psi=\left(\psi_{1}, \psi_{2}, \psi_{3}, \cdots \psi_{n}\right)^{T}$. If $\mathrm{G}$ operator is unitary in the Hilbert space, subject to the theory of Beniof's quantum Turing machine, the single step operator $\mathrm{H}$ can be constructed according to operator $\mathrm{G}$, that is:

$$
\psi(t)=H^{t}\left(\psi\left(x_{0}, 0\right)\right)=H\left(\cdots H\left(\psi\left(x_{0}, 0\right)\right) \cdots\right),
$$

$\operatorname{Here} \psi\left(x_{0}, 0\right)$ is the initial state.

\section{The study algorithm for quantum perception machine}

We suppose that a quantum neuron network (QNN) can accept $n$ imports $\left(\left|x_{1}>,\right| x_{2}>,\left|x_{2}>, \cdots\right| x_{n}>\right)$ simultaneously, the output $\mid y(t)>$ of each neuron will change with time. $\mid y(t)>$ can be defined as follows:

$$
\begin{gathered}
\left|y(t)>=(-1)^{f(x)} \cdot \sum_{j=1}^{n} \omega(t)_{i}\right| x_{j}>, \\
f(x)= \begin{cases}1 & y=d \\
0 & y \neq d\end{cases}
\end{gathered}
$$

Here, $f(x)$ is double-valued function, $\hat{\omega}_{j}$ is $2 \times 2$ Pauli matrix and is constructed by the qubit $\{|0>| 1>\},$,$d is$ the desired output. It is known from the analysis of Section III, it is $3 / 4$ for the probability which the actual output of a neuron is not equal to the desired output. $|y\rangle$ is the single quantum state, and here select

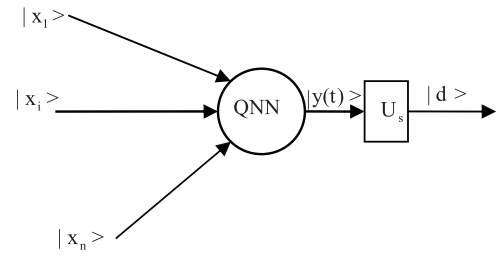

Fig. 1 The quantum neural model.

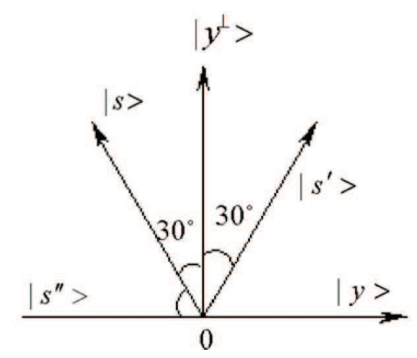

Fig. 2 Rotation images of two-qubit.

$\mid y>=(|0>-| 1>) / \sqrt{2}$. Associated with Equation (9), a reflection can be constituted by taking $\mid s>$ :

$$
U_{s}=2|s><s|-1,
$$

It makes the states $\mid s>$ keep invariant but can reverse any symbol with the orthogonal states. It keeps the component of form $\mid s>$ unchangeable on geometry, but changes the component symbol on the perpendicular hyperplane with state $\mid s>$.

If the system output is $|y\rangle$, as there are 4 kinds of possibilities in the mutual interaction between the neuron and the environment, $N=4$ :

$$
<y \mid s>=\frac{1}{\sqrt{N}}=\sin \theta=\frac{1}{2}
$$

$\theta=30^{\circ}, 2 \theta=60^{\circ}$. Figure 2 shows that $U_{s}$ rotates the state $\mid s>$ and $\mid y>$ to the same straight line after doing one iteration.

It can be sure to draw the desired state $\mid y>$ when $U_{s}$ project on the calculation base and do the measurement. This indicates that the quantum neuron possesses the ability for learning to know in one time.

\section{The example of study}

In order to demonstrate the effectiveness of the algorithm, we give out a simple example. Supposed the desired output of a quantum neuron is $|d\rangle=(1,1)^{T}$, the output probability amplitude and the average probability 


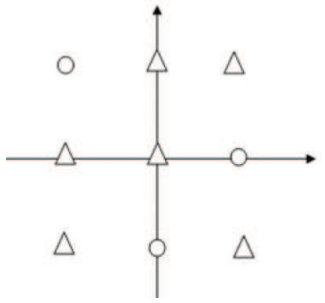

Fig. 3 The nine-point pattern distribution map.

amplitude are both $1 / 2$ the quantum network output without training is one kind of the four possibilities:

$$
\left\{I \otimes\left|d>, \sigma_{1} \otimes\right| d>\otimes, \sigma_{2}\left|d>, \sigma_{3} \otimes\right| d>\right\}
$$

The neuron study course is as below:

(1) Initializing the quantum state to

$$
\left[\mid d>\frac{1}{\sqrt{2}}(|0>-| 1>)\right]
$$

(2) It changes the symbol of probability amplitude of the state $|d\rangle$ through the effect of symbol factor $f(x)$, from $1 / 2$ to $-1 / 2$, thus the original $1 / 2$ of average probability amplitude reduces to $1 / 4$.

(3) After passing the effect of $U_{s}$, the probability amplitude of other circumstances changes into $2 \times \frac{1}{4}-\frac{1}{2}=0$, and the probability amplitude of the desired output state $\mid d>$ changes into $2 \times \frac{1}{4}-\left(-\frac{1}{2}\right)=1$.

In other words, the quantum neuron can learn in one time to get the desired output. To test QNN algorithm performance, we choose simulation of typical examples of pattern recognition and Common Back Propagation algorithm (CBP) for performance comparison.

Nine samples of pattern recognition as shown in Figure 3, This mode is a typical two-class classification problem, which can be seen as "exclusive or" generalization of the problem, often as the inspection algorithms of classification ability scale. By CBP and QNN, respectively as a classifier, network structure are taken by a 2-10-1 model, limited number of iteration step is 15,000 , error limited precision of 0.01 , learning rate from $0.1,0.2,0 \ldots 1$ in the value. For each learning rate, respectively QNN and CBP to 100 times the simulation, convergence times were recorded as the evaluation index, such as the maximum number of Iterative steps, the minimum number of Iterative steps, and the average number of iterative steps.etc. The learning rate is changed, as the comparison of the convergence of the two models shown in Figure 4, and the comparison of the number of Iterative step shown in Figure 5.

Figure 4 shows that when learning rate changes, QNN convergence rate of $100 \%$, while the CBP convergence rate of the minimum $22 \%$, Max was only $69 \%$. Figure 5 shows that when learning rate changes, QNN, the average

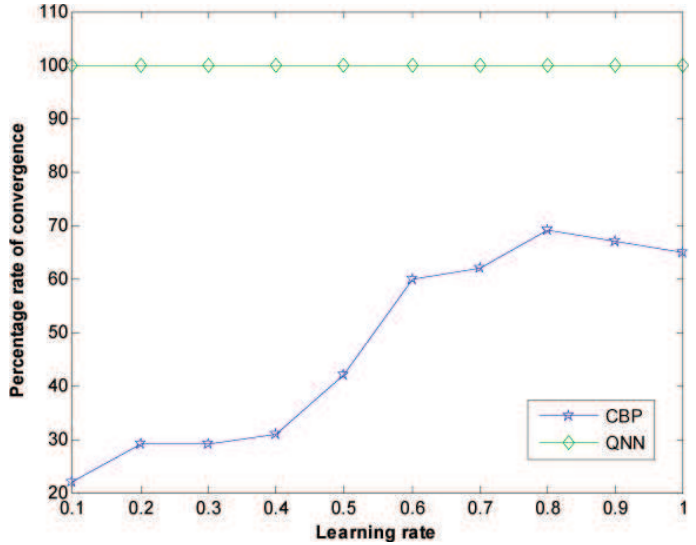

Fig. 4 QNN and CBP convergence rate.

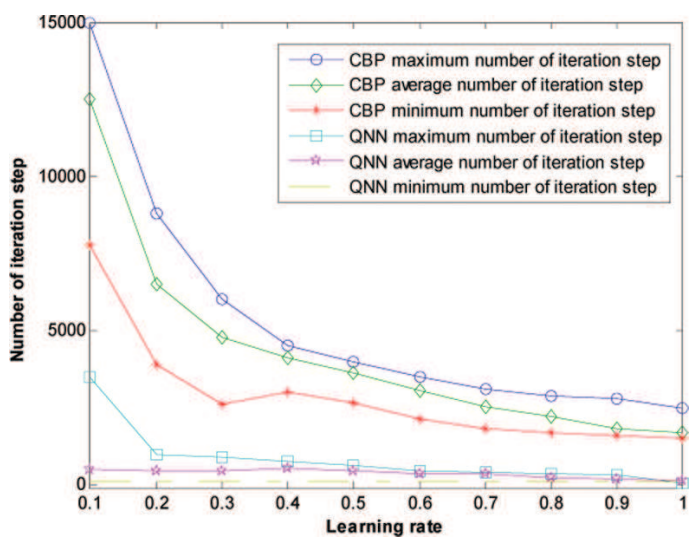

Fig. 5 QNN and CBP number of iterative step.

number of Iterative steps up to 687.70 , down to 275.01 , fluctuation range of only 412.69. Average number of CBP iteration steps up to 12335.45 , about 6 times of QNN, and fluctuation range is up to 10638.21, about 26 times of QNN. Simulation results show that QNN is not only a small number of iteration step, high rate of convergence, when the parameters change with strong robustness.

\section{Conclusions}

At present, although study of quantum neural computing is still in its infancy, and the theory is far from mature, there are theoretical analyses and application which have proven that quantum neural computing (QNC), compared with the traditional neural computing, has significant advantages in the following areas:

-exponential memory capacity and memory speed;

-the smaller network size and simple network topology; -improved stability and effectiveness; 
-fast learning, a learning and high-speed processing of information;

-To eliminate the potential for catastrophic loss of memory

-Eliminate the potential for catastrophic memory loss and so on.

These advantages are for people to create a large-capacity, high-speed information processing system which provides new and superior information processing possible capacity, and helps people re-understand intelligence and brain function.

\section{Acknowledgements}

This work was supported by the Natural Science Foundation of China under Grant No.10902125

\section{References}

[1] M.V. Altaisky. Quantum neural network. Technical report, http://arxiv.org/PS_cache/quant-ph/pdf/0107/0107012.pdf, 2001.

[2] R. Beale and T. Jackson. Neural Computing. MIT Press, 1994.

[3] E. Behrman, L. Nash, J. Steck, V. Chandrashekar, and S. Skinner. Simulations of quantum neural networks. Information Sciences, 128(3-4):257-269, October 2000.

[4] E. Behrman, J. Niemel, and J. Steck. A quantum dot neural network. Technical report, Www.engr.twsu.edu/ me/faculty/steck/pubs/ieeenn96.ps, 1996.

[5] S. Gupta and R. Zia. Quantum neural networks. Technical report, http://www.arxiv.org/PS_cache/quant-ph/pdf/ 0201/0201144.pdf, 2002.

[6] Rigui Zhou, "Quantum Competitive Neural Network", International Journal of Theoretical Physics, vol. 49, no. 1, (2010)110-119,

[7] Wei Sun, Yu Jun He, Ming Meng, "A Novel Quantum Neural Network Model with Variable Selection for Short Term Load Forecasting", Applied Mechanics and Materials , vol. 20-23, (2010)612-617,

[8] G. Y. Lian, K. L. Huang, J. H. Chen, F. Q. Gao, Training algorithm for radial basis function neural network based on quantum-behaved particle swarm optimization, International Journal of Computer Mathematics-IJCM, vol. 87, no. 3, (2010)629-641,

[9] Panchi Li, Kaoping Song, Erlong Yang, Quantum genetic algorithm and its application to designing fuzzy neural controller, International Conference on Natural Computation-ICNC, (2010)2994-2998,

[10] Christopher Altman, Romàn R. Zapatrin, "Backpropagation training in adaptive quantum networks", International Journal of Theoretical Physics, 49, 2991 (2010)

\section{Yanhua}

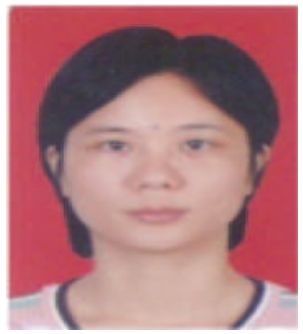

Zhong Guangdong Province, China. Birth date: October, 1974, is Software Engineering M.S., graduated from Computer College, Guangdong University of Technology, China. And research interests on intelligent algorithms and quantum computing. She is an associate professor of Jiangmen

Polytechnic.

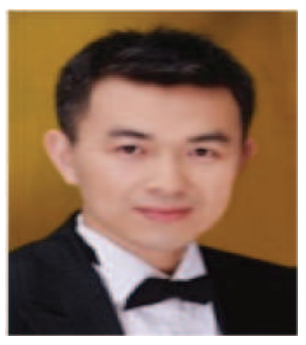

\section{Changqing}

Yuan

Jilin Province, China. Birthday: October, 1974. is Mechanical Engineering Ph.D., graduated from School of Aerospace, Tsinghua University. And research interests on Spacecraft dynamics and Control. He is an associate professor of Aviation University of Air Force. 